Internist 2018· 59:113

https://doi.org/10.1007/s00108-017-0374-1

Online publiziert: 11. Januar 2018

(c) Springer Medizin Verlag GmbH, ein Teil von Springer Nature 2018

CrossMark

\author{
W. Hiddemann ${ }^{1} \cdot$ M. Reincke ${ }^{2}$ \\ 'Medizinische Klinik und Poliklinik III, Klinikum der Universität München, Ludwig-Maximilians-Universität \\ München, München, Deutschland \\ ${ }^{2}$ Medizinische Klinik und Poliklinik IV, Klinikum der Universität München, Ludwig-Maximilians-Universität \\ München, München, Deutschland
}

\title{
Paraneoplastische Syndrome
}

Unter dem Begriff der paraneoplastischen Syndrome werden Begleitsymptome einer Krebserkrankung zusammengefasst, die nicht primär durch den Tumor selbst bedingt sind. Sie treten bei etwa 5-15\% aller Krebserkrankungen auf und können der Diagnose eines malignen Tumors teils längere Zeit vorausgehen. Die zugrunde liegenden Mechanismen sind unterschiedlich. Sie können etwa in der Produktion von Zytokinen oder hormonähnlichen Substanzen, aber auch in zellulären oder humoralen Immunreaktionen bestehen.

Bei den paraneoplastischen Syndromen (PNS) werden entsprechend ihrer klinischen Manifestation sechs Hauptgruppen unterschieden:

- Endokrine PNS

- Neurologische PNS

- Muskuloskeletale und rheumatische PNS

- PNS der Haut

- Hämatologische PNS

- PNS, die sich diesen Gruppen nicht zuordnen lassen

Kenntnisse über PNS sind für jeden Internisten von erheblicher Relevanz: Einerseits stellen sich in der Praxis viele Patienten mit auf den ersten Blick unspezifischen Symptomen vor, andererseits leiden viele unter Beschwerden, die nicht primär an eine Tumorerkrankung denken lassen. Daher werden PNS häufig verkannt, was zu später Diagnosestellung und gravierenden Komplikationen führen kann. Gleichzeitig besteht bei einigen PNS die Chance auf eine frühe Diagnose der zugrunde liegenden Tumorerkrankung, wenn diese bisher okkult war.

In der vorliegenden Ausgabe von Der Internist werden die Hauptgruppen der neoplastischen Syndrome von hoch kompetenten Kolleginnen und Kollegen anschaulich vorgestellt. Die Autoren geben auch wertvolle Hinweise für den klinischen Alltag. So bieten S. Krug u. P. Michl einen Überblick über metabolische Entgleisungen als Ausdruck eines PNS und ergänzen damit die Ausführungen von N. Reisch und dem Mitherausgeber zu endokrin bedingten PNS, wie dem ektopen Cushing-Syndrom und der Hyponatriämie bzw. dem Syndrom der inadäquaten Sekretion von antidiuretischem Hormon (SIADH). Dass PNS auch an der Haut, an Gelenken und Weichteilen manifest werden können, unterstreichen die Beiträge von C. Gießen-Jung, A. Wollenberg und M. Reinholz bzw. J. Leipe und H. Schulze-Koops. Ein großer Teil der PNS äußert sich in neurologischen Symptomen, die von D. De Simoni und R. Höftberger diskutiert werden.

\section{\) Paraneoplastische Syndrome werden häufig verkannt}

Insgesamt gibt der vorliegende Schwerpunkt einen umfassenden Überblick über die Breite und Komplexität paraneoplastischer Syndrome. Damit bietet er wertvolle Informationen für jeden Internisten, ob nun in Weiterbildung oder mit Facharztexpertise. Wir hoffen somit, eine informative Ausgabe zusammengestellt zu haben, und wünschen unseren Lesern eine gute Lektüre.



Prof. Dr. W. Hiddemann

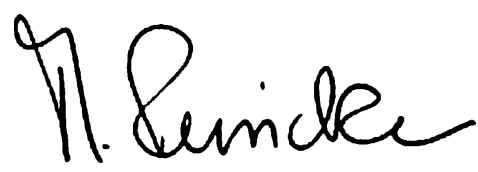

Prof. Dr. M. Reincke

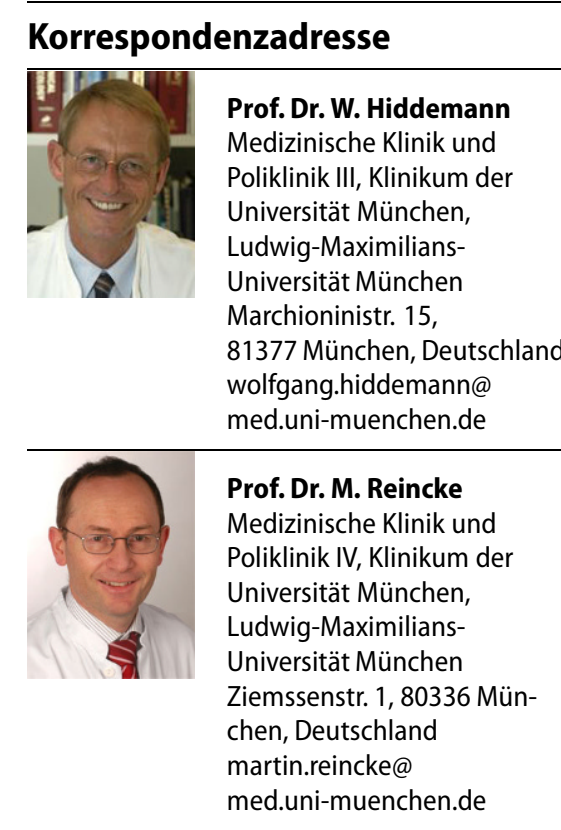

Interessenkonflikt. W. Hiddemann und M. Reincke geben an, dass kein Interessenkonflikt besteht. 